\title{
THE ENVELOPE OF HOLOMORPHY OF RIEMANN DOMAINS OVER A COUNTABLE PRODUCT OF COMPLEX PLANES
}

\author{
BY \\ MÁRIO C. MATOS
}

\begin{abstract}
This paper deals with the problem of constructing envelopes of holomorphy for Riemann domains over a locally convex space. When this locally convex space is a countable product of complex planes the existence of the envelope of holomorphy is proved and the domains of holomorphy are characterized.
\end{abstract}

For the Riemann domains over the cartesian product $\boldsymbol{C}^{\boldsymbol{N}}$ of a countable number of complex planes, the domains of holomorphy are characterized and the existence of the envelope of holomorphy is proved. Also, for Riemann domains over a complex separated locally convex space $E$ such that the closed convex hull of every compact subset is compact, the existence of the normal envelope of holomorphy is proved.

Let $(\boldsymbol{U}, \varphi)$ be a Riemann domain over $E$. This means that $\boldsymbol{U}$ is a connected separated topological space and $\varphi$ is a local homeomorphism from $\boldsymbol{U}$ into $E$. If $\boldsymbol{a} \in \boldsymbol{U}$ and $A \subset E, \boldsymbol{a}+A$ is defined by

$$
\boldsymbol{a}+A=[\varphi \mid \boldsymbol{W}]^{-1}(\varphi(\boldsymbol{a})+A),
$$

$\boldsymbol{W}$ being an open subset of $\boldsymbol{U}$ where $\varphi$ is a homeomorphism, $\boldsymbol{a} \in \boldsymbol{W}$, and $\varphi(\boldsymbol{a})+\boldsymbol{A}$ $\subset_{\varphi}(\boldsymbol{W})$. When $A$ has only one element $h, \boldsymbol{a}+h$ denotes the unique element of $\boldsymbol{a}+\{h\}$. If $\boldsymbol{B}$ is a subset of $\boldsymbol{U}$,

$$
\boldsymbol{B}+A=\bigcup_{\boldsymbol{b} \in \boldsymbol{B}}(\boldsymbol{b}+A)
$$

where $\boldsymbol{b}+A$ has the meaning just stated. A complex mapping $f$ defined in $\boldsymbol{U}$ is holomorphic if, for every $\boldsymbol{u}$ in $\boldsymbol{U}$, there is an open convex balanced neighborhood $U$ of zero in $E$ and a sequence of continuous $n$-homogeneous polynomials in $E$ : $(n !)^{-1} \hat{d}^{n} f(\boldsymbol{u}), n=0,1, \ldots$, such that $\boldsymbol{u}+U \subset \boldsymbol{U}$ and

$$
f(\boldsymbol{u}+h)=\sum_{n=0}^{\infty}(n !)^{-1} \hat{d}^{n} f(\boldsymbol{u})(h),
$$

the series converging uniformly for $h$ in $U$. In the algebra $\mathscr{H}(\boldsymbol{U})$ of all complex

Received by the editors July 6,1971 .

AMS 1970 subject classifications. Primary 46A99; Secondary 58B10.

Key words and phrases. Holomorphic mappings, domains of holomorphy, normal envelope of holomorphy, envelope of holomorphy, Riemann domain over a locally convex space.

Copyright (C) 1972, American Mathematical Society 
holomorphic mappings in $\boldsymbol{U}$ it is considered the Nachbin topology generated by all algebra seminorms ported by compact subsets of $\boldsymbol{U}[1]$. Let $S(\boldsymbol{U})$ be the spectrum of $\mathscr{H}(\boldsymbol{U})$, that is, the set of all continuous homomorphisms from $\mathscr{H}(\boldsymbol{U})$ onto $\boldsymbol{C}$.

Proposition 1. If $h \in S(U)$, there is a unique $a_{h} \in E$ such that $T\left(a_{h}\right)=h(T \circ \varphi)$ for all $T$ in the topological dual $E^{\prime}$ of $E$.

Proof. There is a compact subset $\boldsymbol{K}$ of $\boldsymbol{U}$ such that

$$
|h(f)| \leqq \sup \{|f(\boldsymbol{k})| ; \boldsymbol{k} \in \boldsymbol{K}\}
$$

for every $f$ in $\mathscr{H}(\boldsymbol{U})$. This fact is denoted by $h \prec \boldsymbol{K}$. Let $K$ be the closed convex hull of $\varphi(K)$. If $\mathscr{T}=\left\{T_{1}, T_{2}, \ldots, T_{n}\right\}$ is a finite subset of $E^{\prime}$ and

$$
\alpha_{\mathscr{T}}=\left\{x \in K ; T_{i}(x)=h\left(T_{i} \circ \varphi\right), i=1,2, \ldots, n\right\},
$$

$\alpha_{\mathscr{T}}$ is nonempty. If this was not the case, $T=\left(T_{1}, \ldots, T_{n}\right)$ would be a continuous mapping from $E$ into $C^{n}$ such that $\left(h\left(T_{1} \circ \varphi\right), \ldots, h\left(T_{n} \circ \varphi\right)\right) \notin T(K)$. Hence there would be a complex linear mapping $G$ in $C^{n}$ such that

$$
\operatorname{Re} G\left(h\left(T_{1} \circ \varphi\right), \ldots, h\left(T_{n} \circ \varphi\right)\right)>\sup \{\operatorname{Re} G \circ T(x) ; x \in K\} .
$$

This would imply that the holomorphic mapping in $U f=\exp G \circ T \circ \varphi$ would satisfy the inequality

$$
|h(f)|>\sup \{|f(\boldsymbol{u})| ; \boldsymbol{u} \in \boldsymbol{K}\},
$$

against the assumption that $h \prec K$. Since $K$ is compact and the collection $\left\{\alpha \mathscr{T} ; \mathscr{T} \subset E^{\prime}\right.$, $\mathscr{T}$ finite $\}$ of closed subsets of $K$ satisfies the finite intersection property, the intersection of the whole collection is nonempty. This intersection has a unique point because $E^{\prime}$ separates the points of $E$.

Let $h \prec K$ and $U$ be an open balanced convex neighborhood of zero in $E$ such that $\boldsymbol{K}+U \subset \boldsymbol{U}$ and $\boldsymbol{K}+L$ is compact for every compact subset $L$ of $U$. If $u \in U$ and $f \in \mathscr{H}(\boldsymbol{U})$

$$
h_{u}(f)=\sum_{n=0}^{\infty}(n !)^{-1} h\left(\hat{d}^{n} f(\cdot)(u)\right)
$$

defines an element $h_{u}$ of $S(\boldsymbol{U})$ such that $a_{h_{u}}=a_{h}+u$. For all $h, \boldsymbol{K}$ and $U$ as above, the collection of all

$$
N_{h, U}=\left\{h_{u} ; u \in U\right\}
$$

defines a topology in $S(\boldsymbol{U})$ such that the mapping $\pi: h \in S(\boldsymbol{U}) \mapsto a_{h} \in E$ is a local homeomorphism which maps the connected open set $N_{h, U}$ onto $\pi(h)+U$ homeomorphically. The proofs of these facts are the same as those of the case in which $E$ is a Banach space [2]. It is supposed now that $\mathscr{H}(\boldsymbol{U})$ separates the points of $\boldsymbol{U}$. If $i(\boldsymbol{u}) \in S(\boldsymbol{U})$ is the evaluation homomorphism associated to $\boldsymbol{u} \in \boldsymbol{U}$, then $i: \boldsymbol{U} \rightarrow S(\boldsymbol{U})$ is a biholomorphism from $\boldsymbol{U}$ onto an open subset $\boldsymbol{U}_{S}$ of $S(\boldsymbol{U})$. Let $(E(\boldsymbol{U}), \pi)$ be the Riemann domain over $E$, where $E(U)$ is the connected component of $S(U)$ containing $\boldsymbol{U}_{S}$. If $f \in \mathscr{H}(\boldsymbol{U})$, its extension $f^{\prime} \in \mathscr{H}(E(\boldsymbol{U}))$ is defined by $f^{\prime}(h)=h(f)$ for all $h$ in $E(\boldsymbol{U})$. 
Let $(\boldsymbol{E}, \psi)$ and $(\boldsymbol{D}, \varphi)$ be Riemann domains over $E$ such that $\boldsymbol{D}$ is canonically identified to an open subset of $\boldsymbol{E}$ by means of a biholomorphism. $(\boldsymbol{E}, \boldsymbol{D})$ is an extension pair if for each $f$ in $\mathscr{H}(\boldsymbol{D})$ there is $f^{\prime} \in \mathscr{H}(\boldsymbol{E})$ such that $f^{\prime} \mid \boldsymbol{D}=f$. If the mapping $f \in \mathscr{H}(\boldsymbol{D}) \mapsto f^{\prime} \in \mathscr{H}(\boldsymbol{E})$ is a homeomorphism, the extension pair $(\boldsymbol{E}, \boldsymbol{D})$ is normal.

$(E(U), U)$ is an extension pair. It is normal by the following result.

THEOREM 1. Let $(\boldsymbol{E}, \boldsymbol{D})$ be an extension pair. If, for every $\boldsymbol{x}$ in $\boldsymbol{E}$, the mapping $f \in \mathscr{H}(\boldsymbol{D}) \mapsto f^{\prime}(\boldsymbol{x}) \in \boldsymbol{C}$ is linear and continuous, then $(\boldsymbol{E}, \boldsymbol{D})$ is normal.

Proof. Let $\pi$ and $\varphi$ be the local homeomorphisms defining the Riemann domains $\boldsymbol{E}$ and $\boldsymbol{D}$ respectively. To prove the theorem it is enough to show that for every algebra seminorm $p$ in $\mathscr{H}(\boldsymbol{E})$, ported by a compact subset $\boldsymbol{L}$ of $\boldsymbol{E}$, there is an algebra seminorm $q$ in $\mathscr{H}(D)$, ported by some compact subset $\boldsymbol{K}$ of $\boldsymbol{D}$, such that $p\left(f^{\prime}\right) \leqq q(f)$ for all $f$ in $\mathscr{H}(\boldsymbol{D})$. By the assumptions of the theorem, for each $\boldsymbol{x}$ in $\boldsymbol{L}$ there is a compact subset $\boldsymbol{K}_{\boldsymbol{x}}$ of $\boldsymbol{D}$ such that $\left|f^{\prime}(\boldsymbol{x})\right| \leqq \sup \left\{|f(\boldsymbol{t})| ; \boldsymbol{t} \in \boldsymbol{K}_{\boldsymbol{x}}\right\}$ for every $f$ in $\mathscr{H}(\boldsymbol{D})$. Let $V_{\boldsymbol{x}}$ be a closed convex balanced neighborhood of zero in $E$ such that $V_{x}$ is contained in the interior $\left(2 V_{x}\right)^{\circ}$ of $2 V_{x}$ and (i) $x+2 V_{x} \subset \boldsymbol{E}$, (ii) $\boldsymbol{K}_{x}+4 V_{x} \subset \boldsymbol{D}$, (iii) $K_{x}+L$ is compact for all compact subsets $L$ of $4 V_{x}$. Since $L$ is compact, there is a finite cover $\left\{\boldsymbol{x}_{i}+V_{\boldsymbol{x}_{i}}=\boldsymbol{x}_{i}+V_{i} ; i=1,2, \ldots, n\right\}$ of $\boldsymbol{L}$. For $i=1,2, \ldots, n$ we set:

$$
\boldsymbol{L}_{i}=\boldsymbol{K}_{\boldsymbol{x}_{i}}+\bigcup_{|\lambda| \leqq 2} \lambda\left\{\left[\pi\left(\boldsymbol{L} \cap\left(\boldsymbol{x}_{i}+V_{i}\right)\right)-\pi\left(\boldsymbol{x}_{i}\right)\right] \cap V_{i}\right\} .
$$

Hence $\boldsymbol{L}_{i} \subset \boldsymbol{K}_{\boldsymbol{x}_{i}}+2 V_{i} \subset \boldsymbol{D}$ and $\boldsymbol{L}_{i}$ is compact. Let $\boldsymbol{K}$ be the union of the $\boldsymbol{L}_{i}, i=1,2$, $\ldots, n$, which is a compact subset of $\boldsymbol{D}$. For every open subset $\boldsymbol{W}$ of $\boldsymbol{D}$ containing $\boldsymbol{K}$, there is an open balanced neighborhood $A$ of zero in $E$ such that

$$
\boldsymbol{W}_{i}=\boldsymbol{K}_{\boldsymbol{x}_{i}}+\bigcup_{|\lambda| \leq 2} \lambda\left\{\left[\pi\left((\boldsymbol{L}+A) \cap\left(\boldsymbol{x}_{i}+\left(2 V_{i}\right)^{\circ}\right)\right)-\pi\left(\boldsymbol{x}_{i}\right)\right] \cap\left(2 V_{i}\right)^{\circ}\right\}
$$

is an open subset of $\boldsymbol{D}$ containing $\boldsymbol{L}_{i}$ for all $i=1,2, \ldots, n$. The union of all $\boldsymbol{W}_{i}$ contains $K$. The set $A$ may be chosen in such a way that this union is a subset of $W$. Now

$$
B(\boldsymbol{W})=\bigcup_{i=1}^{n}\left[(\boldsymbol{L}+A) \cap\left(\boldsymbol{x}_{i}+\left(2 V_{i}\right)^{\circ}\right)\right]
$$

is an open subset of $\boldsymbol{E}$ containing $\boldsymbol{L}$. If $\boldsymbol{x} \in B(\boldsymbol{W})$, then $\boldsymbol{x}$ is in $\boldsymbol{x}_{i}+\left(2 V_{i}\right)^{\circ}$ for some $i$, $\pi(\boldsymbol{x})-\pi\left(\boldsymbol{x}_{i}\right)$ belongs to $\left(2 V_{i}\right)^{\circ}$ and, for $|\lambda| \leqq 1, \boldsymbol{x}_{i}+\lambda\left(\pi(\boldsymbol{x})-\pi\left(\boldsymbol{x}_{i}\right)\right)$ is in $\boldsymbol{E}$. The function $g(\lambda)=f^{\prime}\left(x_{i}+\lambda\left(\pi(x)-\pi\left(x_{i}\right)\right)\right)$ defined in a neighborhood of the set

is such that:

$$
\{\lambda \in C ;|\lambda| \leqq 1\}
$$

$$
g(\lambda)=\sum_{m=0}^{\infty} \lambda^{m}(m !)^{-1} g^{(m)}(0) \quad \text { and } \quad f^{\prime}(\boldsymbol{x})=g(1)=\sum_{m=0}^{\infty}(m !)^{-1} g^{(m)}(0) .
$$

Therefore

$$
\begin{aligned}
\left|f^{\prime}(\boldsymbol{x})\right| & \leqq \sum_{m=0}^{\infty}(m !)^{-1}\left|\hat{d}^{m} f^{\prime}\left(\boldsymbol{x}_{i}\right)\left(\pi(\boldsymbol{x})-\pi\left(\boldsymbol{x}_{i}\right)\right)\right| \\
& \leqq \sum_{m=0}^{\infty}(m !)^{-1} \sup _{\boldsymbol{k} \in \boldsymbol{K}_{\boldsymbol{x}_{i}}}\left|\hat{d}^{m} f(\boldsymbol{k})\left(\pi(\boldsymbol{x})-\pi\left(\boldsymbol{x}_{i}\right)\right)\right|
\end{aligned}
$$


If $\boldsymbol{k} \in \boldsymbol{K}_{\boldsymbol{x}_{i}}$, then $\boldsymbol{k}+\lambda\left(\pi(\boldsymbol{x})-\pi\left(\boldsymbol{x}_{i}\right)\right)$ is in $\boldsymbol{D}$ for all $|\lambda| \leqq 2$. The function $g_{1}(\lambda)=$ $f\left(\boldsymbol{k}+\lambda\left(\pi(\boldsymbol{x})-\pi\left(\boldsymbol{x}_{i}\right)\right)\right)$ is defined for all $|\lambda| \leqq 2$. The Cauchy inequalities for this function at 0 imply that

$$
\left|\hat{d}^{m} f(\boldsymbol{k})\left(\pi(\boldsymbol{x})-\pi\left(\boldsymbol{x}_{\boldsymbol{i}}\right)\right)\right| \leqq m ! 2^{-m} \sup _{\boldsymbol{t} \in W_{\boldsymbol{i}}}|f(\boldsymbol{t})|
$$

for $m=0,1,2, \ldots$ These inequalities and (1) imply

$$
\left|f^{\prime}(x)\right| \leqq \sup _{t \in W_{i}}|f(t)| \cdot \sum_{m=0}^{\infty} 2^{-m} \leqq 2 \sup _{t \in W}|f(t)| .
$$

Therefore

$$
\sup _{\boldsymbol{x} \in B(\boldsymbol{W})}\left|f^{\prime}(\boldsymbol{x})\right| \leqq 2 \sup _{\boldsymbol{t} \in \boldsymbol{W}}|f(\boldsymbol{t})|
$$

for every open subset $\boldsymbol{W}$ of $\boldsymbol{D}$ such that $\boldsymbol{K}$ is contained in $\boldsymbol{W}$. It follows that for every open subset $\boldsymbol{W}$ of $\boldsymbol{D}$ containing $\boldsymbol{K}$ there is $c(B(\boldsymbol{W}))>0$ such that

$$
p\left(f^{\prime}\right) \leqq 2 c(B(\boldsymbol{W})) \sup _{\boldsymbol{t} \in \boldsymbol{W}}|f(\boldsymbol{t})|
$$

for all $f$ in $\mathscr{H}(\boldsymbol{D})$. The supremum of the family of all algebra seminorms $s$ in $\mathscr{H}(\boldsymbol{D})$, ported by $K$ and such that

$$
s(f) \leqq 2 c(B(\boldsymbol{W})) \sup _{\boldsymbol{t} \in \boldsymbol{W}}|f(\boldsymbol{t})|
$$

for all open sets $\boldsymbol{W}$ containing $\boldsymbol{K}$ and all $f$ in $\mathscr{H}(\boldsymbol{D})$, is a member of this family. This supremum is the seminorm $q$ we need to complete the proof of this theorem.

Now it is easy to show the following result:

Proposition 2. If $(\boldsymbol{U}, \varphi)$ is a Riemann domain over $E$ such that $\mathscr{H}(\boldsymbol{U})$ separates the points of $\boldsymbol{U}$, then $(E(U), \pi)$ and the biholomorphism i from $\boldsymbol{U}$ onto the open subset $U_{S}$ of $E(U)$ are such that (a) $\mathscr{H}(E(U))$ separates the points of $E(U) ;(b)(E(U), U)$ is a normal extension pair. Moreover, $(E(U), \pi)$ is maximum relative to (a) and (b) in the following sense: if $(\boldsymbol{M}, \psi)$ is a Riemann domain over $E$ and $j$ is a biholomorphism from $\boldsymbol{U}$ onto an open subset $\boldsymbol{U}_{\boldsymbol{M}}$ of $\boldsymbol{M}$ and (a) and (b) are satisfied when we replace $E(\boldsymbol{U})$ by $\boldsymbol{M}$, then $\boldsymbol{M}$ may be identified to an open subset of $E(\boldsymbol{U})$ by a biholomorphism preserving the points of $\boldsymbol{U}$.

Throughout the remaining part of this paper $E=C^{N}$ and the points and the subsets of $C^{n}$ are identified to the points and subsets of $C^{n} \times(0,0, \ldots) \subset C^{N}$, $n=1,2, \ldots$

A Riemann domain $(\boldsymbol{U}, \varphi)$ over $\boldsymbol{C}^{\boldsymbol{N}}$ is of order $n$ at a point $\boldsymbol{u}$ of $\boldsymbol{U}$ if $n$ is the smallest positive integer such that there is an open polydisc $B$ in $\pi_{n}^{-1}\left(C^{N}\right)$ with center 0 for which $\boldsymbol{u}+v \in \boldsymbol{U}$ for every $v$ in $\pi_{n}^{-1}(B)$. Here $\pi_{n}$ denotes the projection mapping from $C^{N}$ onto the space of the first $n$ variables. $(U, \varphi)$ is of order (at most) $n$ in a subset $\boldsymbol{A}$ of $\boldsymbol{U}$ if $(\boldsymbol{U}, \varphi)$ is of order (at most) $n$ at each point of $\boldsymbol{A} .(\boldsymbol{U}, \varphi)$ is locally pseudoconvex if $\left(\boldsymbol{U}_{V}, \varphi_{V}\right)$ is pseudoconvex for each affine subspace $V$ of $C^{N}$ of dimension two. $\boldsymbol{U}_{V}$ denotes the topological subspace $\varphi^{-1}[\varphi(\boldsymbol{U}) \cap V]$ of $\boldsymbol{U}$ and 
$\varphi_{V}$ denotes the restriction of $\varphi$ to $\boldsymbol{U}_{V}$. In this case, for each $v$ in $V$, the mapping $\boldsymbol{z} \in \boldsymbol{U}_{V} \mapsto-\log \delta_{U_{V}}(\boldsymbol{z}, v) \in \boldsymbol{C}$ is plurisubharmonic. Recall that

$$
\delta_{\boldsymbol{U}_{V}}(\boldsymbol{z}, v)=\inf \left\{|\lambda| ; \boldsymbol{u}+\lambda v \in \boldsymbol{U}_{v}\right\} .
$$

Proposition 3. If $(\boldsymbol{U}, \varphi)$ is a locally pseudoconvex Riemann domain over $\boldsymbol{C}^{\boldsymbol{N}}$, then there is a positive integer $n$ such that $(U, \varphi)$ is of order $n$ in $U$ and

$$
\varphi(\boldsymbol{U})=\pi_{n} \circ \varphi(\boldsymbol{U}) \times C^{N-[0, n-1]} .
$$

LEMMA 1. Let $(\boldsymbol{U}, \varphi)$ be locally pseudoconvex and of order $n$ at a point $\boldsymbol{u}$ of $\boldsymbol{U}$. Let $\boldsymbol{r}$ be the largest real positive number such that $\boldsymbol{u}+b \in \boldsymbol{U}$ for each $b$ in the open polydisc $B_{r}$ in $\pi_{n}\left(C^{N}\right)$ with center 0 and radius $r$. Then $\boldsymbol{u}+v \in \boldsymbol{U}$ for each $v$ in $\pi_{n}^{-1}\left(B_{r}\right)$. $(\boldsymbol{U}, \varphi)$ is of order at most $n$ at each one of these $\boldsymbol{u}+v$.

Proof. $\varphi(\boldsymbol{u})$ may be considered equal to zero with no loss of generality. Let $w=\left(w_{j}\right)_{j \in N} \in \pi_{n}^{-1}\left(B_{r}\right)$. If $\pi_{n}(w)=0$, then $\boldsymbol{u}+w \in \boldsymbol{U}$ because $(\boldsymbol{U}, \varphi)$ is of order $\boldsymbol{n}$ at $\boldsymbol{u}$. If $\pi_{n}(w) \neq 0$ and $w_{j}=0$ for each $j \geqq n, \boldsymbol{u}+w \in \boldsymbol{U}$ because $w \in B_{r}$. If $\pi_{n}(w) \neq 0$ and $w_{j} \neq 0$ for some $j \geqq n$, consider $z=\left(z_{j}\right)_{j \in N}=\left(0, \ldots, 0, w_{n}, w_{n+1}, \ldots\right) \in C^{N}$. The subspace $V$ of $\boldsymbol{C}^{N}$ generated by $z$ and $w$ has dimension two. Since $\left(\boldsymbol{U}_{V}, \varphi \mid \boldsymbol{U}_{V}\right)$ is pseudoconvex, $-\log \delta_{U_{V}}(\boldsymbol{t}, w-z)$ is a plurisubharmonic function of $\boldsymbol{t}$ in $\boldsymbol{U}_{V} \cdot(\boldsymbol{U}, \varphi)$ of order $n$ at $\boldsymbol{u}$ implies that there are positive real numbers $\varepsilon_{0}, \ldots, \varepsilon_{n-1}$ such that $\boldsymbol{u}+v \in \boldsymbol{U}$ for each $v$ in $\boldsymbol{C}^{\boldsymbol{N}}$ such that $\left|v_{i}\right|<\varepsilon_{i}, i=0,1, \ldots, n-1$. Hence $\boldsymbol{u}+\lambda z \in \boldsymbol{U}_{V}$ for each $\lambda \in \boldsymbol{C}$ because $\lambda z_{i}=0, i=0,1, \ldots, n-1$, and $\varphi(\boldsymbol{u}+\lambda z)=\lambda z \in V$. Consequently $-\log \delta_{U_{v}}(u+\lambda z, w-z)$ is a subharmonic function of $\lambda$ in $C$. If $\varepsilon$ is the minimum of the $\varepsilon_{i}, i=0,1, \ldots, n-1$ and $\delta$ is the product of $\varepsilon$ by the inverse of the supremum of the $\left|w_{i}\right|, i=0,1, \ldots, n$, then $\left|\alpha\left(w_{i}-z_{i}\right)\right|<\varepsilon$ for each $|\alpha|<\delta$ and $i=0,1, \ldots, n-1$. It follows that $\boldsymbol{u}+\lambda z+\alpha(w-z)$ is in $\boldsymbol{U}_{V}$ for all $\lambda$ in $\boldsymbol{C}$ and $|\alpha|<\delta$. Thus $-\log \delta_{\boldsymbol{U}_{v}}(\boldsymbol{u}+\lambda z, w-z)$ is a bounded above subharmonic function of $\lambda$ in $\boldsymbol{C}$, hence constant. Since $\delta_{U_{V}}(\boldsymbol{u}+0 z, w-z)>1, \delta_{U_{V}}(\boldsymbol{u}+z, w-z)>1$ and $\boldsymbol{u}+w \in \boldsymbol{U}$.

LEMMA 2. Let $(\boldsymbol{U}, \varphi)$ and $\boldsymbol{u}$ be as in Lemma 1 . Let $\boldsymbol{W}$ be an open connected neighborhood of $\boldsymbol{u}$ such that $\varphi \mid \boldsymbol{W}$ is a homeomorphism from $\boldsymbol{W}$ onto $\varphi(\boldsymbol{u})+A_{0} \times \cdots \times A_{s}$ $\times C^{N-[0, s]}$, where each $A_{i}$ is an open ball in $C$ with center 0 and $s \geqq n-1$. Then $(U, \varphi)$ is of order at most $n$ in $\boldsymbol{W}$ and $\varphi(\boldsymbol{U}) \supset \varphi(\boldsymbol{u})+A_{0} \times \cdots \times A_{n-1} \times C^{N-[0, n-1]}$.

Proof. $\varphi(\boldsymbol{u})$ may be considered equal to 0 without any loss of generality. Let $W_{n}=A_{0} \times \cdots \times A_{n-1}$ and $W_{n}^{\prime}$ be the set of all points $w$ of $W_{n}$ such that $(\boldsymbol{U}, \varphi)$ is of order at most $n$ at $[\varphi \mid W]^{-1}(w)$. By Lemma 1 , if $w \in W_{n}^{\prime}, W_{n}^{\prime}$ contains the largest polydisc of radius $r>0$ with center $w$ which is contained in $W_{n}$. Since $0 \in W_{n}^{\prime}$, it follows that $W_{n}=W_{n}^{\prime}$. If $\boldsymbol{w} \in \boldsymbol{W}, \varphi(w) \in W_{n}^{\prime} \times \boldsymbol{C}^{\boldsymbol{N}-[0, n-1]}$. Hence, applying Lemma 1 for the case $\boldsymbol{u}=\varphi^{-1}\left[\pi_{n} \varphi(\boldsymbol{w})\right]$, it is easy to see that $(\boldsymbol{U}, \varphi)$ is of order at most $n$ at $\boldsymbol{w}=\boldsymbol{u}+\left(\varphi(\boldsymbol{w})-\pi_{n} \varphi(\boldsymbol{w})\right)$.

Proof of Proposition 1. Let $\boldsymbol{V}$ be the set of all points of $\boldsymbol{U}$ where $(\boldsymbol{U}, \varphi)$ is of order at most $n$. By Lemma $2, \boldsymbol{V}$ is open. Let $\left(\boldsymbol{x}_{k}\right)_{k=0}^{\infty}$ be a sequence of points of $\boldsymbol{V}$ converging to $\boldsymbol{x}$ in $\boldsymbol{U}$. Let $\boldsymbol{W}$ be an open connected neighborhood of $\boldsymbol{x}$ in $\boldsymbol{U}$ such 
that $\varphi \mid \boldsymbol{W}$ is a homeomorphism from $\boldsymbol{W}$ onto $\varphi(\boldsymbol{x})+A_{0} \times \cdots \times A_{s} \times \boldsymbol{C}^{\boldsymbol{N}-[0, s]}$, where each $A_{i}$ is an open ball in $\boldsymbol{C}$ with center 0 . Thus $\boldsymbol{x}_{k} \in \boldsymbol{W}$ for $k$ large enough and, by Lemma 2, $(\boldsymbol{U}, \varphi)$ is of order at most $n$ in $\boldsymbol{W}$. Hence $\boldsymbol{x} \in \boldsymbol{V}$ and $\boldsymbol{V}$ is closed in $\boldsymbol{U}$. Since $\boldsymbol{U}$ is connected, $\boldsymbol{V}$ is equal to $\boldsymbol{U}$. Now the remaining part of the proof follows easily.

Proposition 4. Let $(\boldsymbol{U}, \varphi)$ be a locally pseudoconvex Riemann domain over $\boldsymbol{C}^{\boldsymbol{N}}$. There is $n>0$ in $\boldsymbol{N}$ such that $(\boldsymbol{U}, \varphi)$ is of order $n$ in $\boldsymbol{U}$ and $\left(\boldsymbol{U}_{n}, \varphi_{n}\right)$ is a manifold of holomorphy spread over $\boldsymbol{C}^{n}$ if $\boldsymbol{U}_{n}=\varphi^{-1}\left[\pi_{n} \circ \varphi(\boldsymbol{U})\right]$ and $\varphi_{n}=\varphi \mid \boldsymbol{U}_{n}$.

Proof. Proposition 1 implies that there is a positive $n$ in $\boldsymbol{N}$ such that $(\boldsymbol{U}, \varphi)$ is

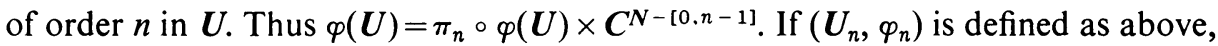
it is a manifold spread over $\boldsymbol{C}^{n}$. Since $(\boldsymbol{U}, \varphi)$ is locally pseudoconvex, $\left(\boldsymbol{U}_{n}, \boldsymbol{\varphi}_{n}\right)$ is locally pseudoconvex, hence a manifold of holomorphy spread over $\boldsymbol{C}^{n}$.

Proposition 5. Let $(\boldsymbol{U}, \varphi)$ be a Riemann domain over $\boldsymbol{C}^{\boldsymbol{N}}$ of order $n$ in $\boldsymbol{U}$ and let $v$ be a point of $\boldsymbol{C}^{N}$. Consider the manifolds spread over $\boldsymbol{C}^{n}\left(\boldsymbol{U}_{n}, \varphi_{n}\right)$ and $\left(\boldsymbol{V}_{n}, \psi_{n}\right)$ given by $\boldsymbol{U}_{n}=\varphi^{-1}\left[\pi_{n} \circ \varphi(\boldsymbol{U})\right], \boldsymbol{V}_{n}=\varphi^{-1}\left[v-\pi_{n}(v)+\pi_{n} \circ \varphi(\boldsymbol{U})\right], \varphi_{n}=\varphi\left|\boldsymbol{U}_{n}, \psi_{n}=\varphi\right| \boldsymbol{V}_{n}$. Then there is a biholomorphism between them. In particular, if $\left(\boldsymbol{U}_{n}, \varphi_{n}\right)$ is of holomorphy, $\left(\boldsymbol{V}_{n}, \psi_{n}\right)$ is also of holomorphy.

Proof. Consider the mappings

$$
\begin{aligned}
& b_{1}: \boldsymbol{x} \in \boldsymbol{V}_{n} \mapsto \boldsymbol{x}+\left[\pi_{n} \circ \varphi(\boldsymbol{x})-\varphi(\boldsymbol{x})\right] \in \boldsymbol{U}_{n}, \\
& b_{2}: \boldsymbol{z} \in \boldsymbol{U}_{n} \mapsto \boldsymbol{z}+\left[v-\pi_{n}(v)\right] \in \boldsymbol{V}_{n} .
\end{aligned}
$$

It is easy to see that $b_{1} \circ b_{2}$ and $b_{2} \circ b_{1}$ are the identity mappings in $\boldsymbol{U}_{n}$ and in $\boldsymbol{V}_{n}$ respectively. They are also local homeomorphisms. In fact consider $\boldsymbol{x}$ in $\boldsymbol{V}_{n}$ and an open neighborhood $\boldsymbol{W}^{\prime}$ of $\boldsymbol{x}$ in $\boldsymbol{U}$ such that $\varphi \mid \boldsymbol{W}^{\prime}$ is a homeomorphism and $\varphi\left(\boldsymbol{W}^{\prime}\right)=\varphi(\boldsymbol{x})+A_{0} \times \cdots \times A_{t} \times \boldsymbol{C}^{\boldsymbol{N}-[0, t]}$, where each $A_{i}$ is an open ball in $\boldsymbol{C}$ with center 0 . Let $\boldsymbol{W}$ be an open neighborhood of $\boldsymbol{x}+\left[\pi_{n} \varphi(\boldsymbol{x})-\varphi(\boldsymbol{x})\right]$ in $\boldsymbol{U}$ such that $\varphi \mid \boldsymbol{W}$ is a homeomorphism and $\varphi(\boldsymbol{W})=\pi_{n} \varphi(x)+B_{0} \times \cdots \times B_{s} \times C^{N-[0, s]}$ is contained in $\pi_{n} \varphi(x)=A_{0} \times \cdots \times A_{t} \times C^{N-[0, t]}$, each $B_{i}$ being an open ball in $C$ with center 0 . Let $\boldsymbol{W}^{\prime \prime}$ be the open neighborhood of $\boldsymbol{x} \in \boldsymbol{U}$ given by

$$
\left[\varphi \mid \boldsymbol{W}^{\prime}\right]^{-1}\left[\varphi(x)+B_{0} \times \cdots \times B_{s} \times C^{N-[0, s]}\right] .
$$

Now it is easy to see that $b_{1} \mid \boldsymbol{W}^{\prime \prime} \cap \boldsymbol{V}_{n}$ is a homeomorphism from $\boldsymbol{W}^{\prime \prime} \cap \boldsymbol{V}_{n}$ onto $\boldsymbol{W} \cap \boldsymbol{U}_{n}$. It is also easy to verify that $f \circ b_{1} \in \mathscr{H}\left(\boldsymbol{V}_{n}\right)$ and $g \circ b_{2} \in \mathscr{H}\left(\boldsymbol{U}_{n}\right)$ for every $f \in \mathscr{H}\left(\boldsymbol{U}_{n}\right)$ and $g \in \mathscr{H}\left(\boldsymbol{V}_{n}\right)$.

A Riemann domain $(\boldsymbol{U}, \varphi)$ over $\boldsymbol{C}^{N}$ is a domain of holomorphy if there is $f \in \mathscr{H}(\boldsymbol{U})$ with no extension $f^{\prime} \in \mathscr{H}\left(\boldsymbol{U}^{\prime}\right)$ for every Riemann domain $\left(\boldsymbol{U}^{\prime}, \varphi^{\prime}\right)$ over $\boldsymbol{C}^{\boldsymbol{N}}$ extending $(\boldsymbol{U}, \varphi)$ properly. $\left(\boldsymbol{U}^{\prime}, \varphi^{\prime}\right)$ extends $(\boldsymbol{U}, \varphi)$ properly if there is a biholomorphism $j$ from $\boldsymbol{U}$ onto a proper open subset $\boldsymbol{U}_{0}$ of $\boldsymbol{U}^{\prime}$. In this case, $f$ has an extension $f^{\prime} \in \mathscr{H}\left(\boldsymbol{U}^{\prime}\right)$ if $f^{\prime} \circ j=f$.

Proposition 6. Let $(\boldsymbol{U}, \varphi)$ be a Riemann domain over $\boldsymbol{C}^{\boldsymbol{N}}$ of order $n$ in $\boldsymbol{U}$ and such that $\left(\boldsymbol{U}_{n}, \varphi_{n}\right)$, defined as above, is a manifold of holomorphy spread over $\boldsymbol{C}^{n}$. Then $(U, \varphi)$ is a domain of holomorphy. 
Proof. There is $f_{n} \in \mathscr{H}\left(\boldsymbol{U}_{n}\right)$ with no extension $f_{n}^{\prime} \in \mathscr{H}\left(\boldsymbol{U}_{n}^{\prime}\right)$ for each manifold $\left(\boldsymbol{U}_{n}^{\prime}, \varphi_{n}^{\prime}\right)$ spread over $\boldsymbol{C}^{n}$ extending $\left(\boldsymbol{U}_{n}, \varphi_{n}\right)$ properly.

$$
f: \boldsymbol{x} \in U \mapsto f(\boldsymbol{x})=f_{n}\left[\boldsymbol{x}+\left(\pi_{n} \varphi(\boldsymbol{x})-\varphi(\boldsymbol{x})\right)\right] \in \boldsymbol{C}
$$

is holomorphic in $\boldsymbol{U}$. In fact: if $\boldsymbol{x}$ is in $\boldsymbol{U}$, let $\boldsymbol{W}^{\prime \prime}$ and $\boldsymbol{W}$ be considered as in the proof of Proposition 5. It is quite clear that

$$
f \circ\left[\varphi \mid \boldsymbol{W}^{\prime \prime}\right]^{-1}(\varphi(\boldsymbol{x})+b)=f \circ[\varphi \mid \boldsymbol{W}]^{-1}\left[\pi_{n} \varphi(\boldsymbol{x})+b\right]
$$

for each $b$ in $B_{0} \times \cdots \times B_{s} \times C^{N-[0, s]}$. But, in $\varphi(\boldsymbol{W}), f \circ[\varphi \mid \boldsymbol{W}]^{-1}$ depends only on the first $n$ variables and it is holomorphic in $\pi_{n} \varphi(\boldsymbol{W})$ since it is equal to

$$
f_{n} \circ\left[\varphi \mid \boldsymbol{W} \cap \boldsymbol{U}_{n}\right]^{-1}
$$

there. Hence it is holomorphic in $\varphi(\boldsymbol{W})([3],[4],[5])$. Since $\varphi(\boldsymbol{W})$ is a translation of $\varphi\left(\boldsymbol{W}^{\prime \prime}\right)$ and $(*)$ holds, $f \circ\left[\varphi \mid \boldsymbol{W}^{\prime \prime}\right]$ is holomorphic in $\varphi\left(\boldsymbol{W}^{\prime \prime}\right)$. Thus $f$ is an element of $\mathscr{H}(\boldsymbol{U})$ and the restriction of it to $\boldsymbol{U}_{n}$ is equal to $f_{n}$. If $f$ has an extension $f^{\prime}$ in $\mathscr{H}\left(\boldsymbol{U}^{\prime}\right)$ for some Riemann domain $\left(\boldsymbol{U}^{\prime}, \varphi^{\prime}\right)$ over $\boldsymbol{C}^{N}$ extending $(\boldsymbol{U}, \varphi)$ properly, there is some manifold $\left(\boldsymbol{V}_{n}, \psi_{n}\right)$ spread over $\boldsymbol{C}^{n}$ (of the type used in the proof of Proposition $5)$ which is not of holomorphy. Proposition 5 implies that $\left(\boldsymbol{U}_{n}, \varphi_{n}\right)$ is not a manifold of holomorphy spread over $\boldsymbol{C}^{n}$, a contradiction to the hypothesis of this proposition. Therefore $(\boldsymbol{U}, \varphi)$ is a domain of holomorphy.

Proposition 7. Let $(\boldsymbol{U}, \varphi)$ be a Riemann domain of holomorphy over $\boldsymbol{C}^{\boldsymbol{N}}$ such that $\mathscr{H}(\boldsymbol{U})$ separates the points of $\boldsymbol{U}$. Then $(E(\boldsymbol{U}), \pi)$ is canonically identified to $(\boldsymbol{U}, \varphi)$.

The proof of this proposition is an immediate consequence of Proposition 2.

A Riemann domain $(\boldsymbol{U}, \varphi)$ over $\boldsymbol{C}^{N}$ is holomorphically convex if, for each compact subset $\boldsymbol{K}$ of $\boldsymbol{U}$ and each balanced convex open neighborhood $U$ of 0 in $\boldsymbol{C}^{\boldsymbol{N}}$ such that $\boldsymbol{K}+U \subset \boldsymbol{U}$ and $\boldsymbol{K}+L$ is compact for every compact subset $L$ of $U$, $\hat{\boldsymbol{K}}_{\boldsymbol{U}}+U \subset \boldsymbol{U}$, where

$$
\hat{\boldsymbol{K}}_{\boldsymbol{U}}=\left\{\boldsymbol{u} \in \boldsymbol{U} ;|f(\boldsymbol{u})| \leqq \sup _{\boldsymbol{t} \in \boldsymbol{K}}|f(\boldsymbol{t})|, \forall f \in \mathscr{H}(\boldsymbol{U})\right\} .
$$

Proposition 8. Let $(\boldsymbol{U}, \varphi)$ be a Riemann domain over $\boldsymbol{C}^{N}$ such that $\mathscr{H}(\boldsymbol{U})$ separates the points of $\boldsymbol{U}$ and $(E(\boldsymbol{U}), \pi)$ is canonically identified to $(\boldsymbol{U}, \varphi)$. Then $(\boldsymbol{U}, \varphi)$ is holomorphically convex.

Proof. Let $\boldsymbol{K}$ be a compact subset of $\boldsymbol{U}$ and let $U$ be an open balanced convex neighborhood of 0 in $C^{N}$ such that $K+U \subset \boldsymbol{U}$ and $\boldsymbol{K}+L$ is compact for every compact subset $L$ of $U$. Then, by the remarks we have done after Proposition 1, $\varphi\left(\hat{\boldsymbol{K}}_{\boldsymbol{U}}\right)+U \subset \varphi(\boldsymbol{U})$ and $N_{i(\boldsymbol{x}), U} \subset S(\boldsymbol{U})$ for each $\boldsymbol{x}$ in $\hat{\boldsymbol{K}}_{\boldsymbol{U}}$. Since $N_{i(\boldsymbol{x}), U}$ is open connected and $\boldsymbol{x}$ is in $E(\boldsymbol{U})$, it follows that $N_{i(\boldsymbol{x}), U}$ is contained in $E(\boldsymbol{U})$ for every $\boldsymbol{x}$ in $\hat{\boldsymbol{K}}_{\boldsymbol{U}}$. But $\boldsymbol{U}$ is identified to $E(\boldsymbol{U})$ and $N_{i(x), U}$ is the same set as $\boldsymbol{x}+U$ for each $\boldsymbol{x}$ in $\hat{\boldsymbol{K}}_{\boldsymbol{U}}$. Hence $\hat{K}_{U}+U \subset \boldsymbol{U}$. 
Proposition 9. If $(\boldsymbol{U}, \varphi)$ is a holomorphically convex Riemann domain over $\boldsymbol{C}^{\boldsymbol{N}}$, it is locally pseudoconvex.

Proof. If $V$ is an affine subspace in $C^{N}$ of dimension two, $\left(\boldsymbol{U}_{V}, \varphi_{V}\right)$ is a manifold spread over $\boldsymbol{C}^{2}$, where $\boldsymbol{U}_{V}=\varphi^{-1}[\varphi(\boldsymbol{U}) \cap V]$ and $\varphi_{V}=\varphi \mid \boldsymbol{U}_{V}$. To show that $\boldsymbol{U}_{V}$ is pseudoconvex, it is enough to prove that $d\left(\hat{\boldsymbol{K}}_{U_{V}}\right)>0$ for every compact subset $\boldsymbol{K}$ of $\boldsymbol{U}_{V}$. Let $\boldsymbol{K}$ be a compact subset of $\boldsymbol{U}_{V}$ and let $U$ be an open balanced convex neighborhood of 0 in $\boldsymbol{C}^{\boldsymbol{N}}$ such that $\boldsymbol{K}+U \subset \boldsymbol{U}$ and $\boldsymbol{K}+L$ is compact for each compact subset $L$ of $U$. Then $\hat{\boldsymbol{K}}_{\boldsymbol{U}}+U \subset \boldsymbol{U}$ and $\varphi\left(\hat{\boldsymbol{K}}_{\boldsymbol{U}}\right)+U \subset \varphi(\boldsymbol{U})$. Since $\varphi\left(\hat{\boldsymbol{K}}_{U}\right)$ is contained in the closed convex hull of $\varphi(K)$ and $\varphi(K)=\varphi_{V}(K) \subset V$, it follows that

$$
\varphi\left(\hat{K}_{U}\right)+U \cap V \subset \varphi(U) \cap V=\varphi_{V}\left(U_{V}\right) .
$$

Now

$$
\hat{\boldsymbol{K}}_{U_{V}}+U \cap V \subset \hat{\boldsymbol{K}}_{U} \cap \boldsymbol{U}_{V}+U \cap V \subset\left(\hat{\boldsymbol{K}}_{U}+U\right) \cap \boldsymbol{U}_{V} \subset \boldsymbol{U} \cap \boldsymbol{U}_{V} \subset \boldsymbol{U}_{V} .
$$

It follows that there is a polydisc $B$ in $V$ with center 0 and radius $r>0$ such that $\hat{\boldsymbol{K}}_{U_{V}}+B \subset \boldsymbol{U}_{V}$. This means that $d\left(\hat{\boldsymbol{K}}_{U_{V}}\right)>0$.

Now it is possible to enunciate the following theorem whose proof we have just finished.

THEOREM 2. Let $(\boldsymbol{U}, \varphi)$ be a Riemann domain over $C^{\boldsymbol{N}}$ such that $\mathscr{H}(\boldsymbol{U})$ separates the points of $\boldsymbol{U}$. The following conditions are equivalent:

(1) $(\boldsymbol{U}, \varphi)$ is a domain of holomorphy.

(2) $(E(U), \pi)$ is canonically identified to $(U, \varphi)$.

(3) $(U, \varphi)$ is holomorphically convex.

(4) $(\boldsymbol{U}, \varphi)$ is locally pseudoconvex.

(5) There is $n>0$ in $N$ such that $(\boldsymbol{U}, \varphi)$ is of order $n$ in $\boldsymbol{U}$ and $\left(\boldsymbol{U}_{n}, \varphi_{n}\right)$ is a manifold of holomorphy spread over $\boldsymbol{C}^{n}$, if $\varphi_{n}=\varphi \mid \boldsymbol{U}_{n}$ and $\boldsymbol{U}_{n}=\varphi^{-1}\left[\pi_{n} \circ \varphi(\boldsymbol{U})\right]$.

REMARKS. (a) Theorem 2 was proved by Hirschowitz in [5] for the case in which $(\boldsymbol{U}, \varphi)$ is an open subset of $\boldsymbol{C}^{N}$.

(b) The implications (1) $\Rightarrow(2) \Rightarrow(3) \Rightarrow(4)$ are true for a Riemann domain $(\boldsymbol{U}, \varphi)$ over a locally convex space $E$ such that the closed convex hull of each compact subset is compact.

Let $(\boldsymbol{U}, \varphi)$ be a Riemann domain over $\boldsymbol{C}^{\boldsymbol{N}}$ such that $\mathscr{H}(\boldsymbol{U})$ separates the points of $\boldsymbol{U}$. The envelope of holomorphy of $(\boldsymbol{U}, \varphi)$ is a Riemann domain $\left(\boldsymbol{U}_{0}, \varphi_{0}\right)$ over $\boldsymbol{C}^{\boldsymbol{N}}$ which is maximum in the sense stated in Proposition 2 with the word "normal" erased in condition (b).

Theorem 2 and Proposition 2 imply that the following result is true:

Proposition 10. If $(U, \varphi)$ is a Riemann domain over $C^{N}$ and $\mathscr{H}(U)$ separates the points of $\boldsymbol{U}$, then $(E(\boldsymbol{U}), \pi)$ is the envelope of holomorphy of $(\boldsymbol{U}, \varphi)$.

\section{REFERENCES}

1. L. Nachbin, Topology on spaces of holomorphic mappings, Ergebnisse der Mathematik und ihrer Grenzgebiete, Band 47, Springer-Verlag, New York, 1969. MR 40 \#7787. 
2. H. Alexander, Analytic functions on Banach spaces, Thesis, University of California, Berkeley, Calif., 1968.

3. C. E. Rickart, Analytic functions of an infinite number of complex variables, Duke Math. J. 36 (1969), 581-597. MR 40 \#7819.

4. J. A. Barroso, Topologias nos espaços de applicações holomorfas entre espaços localmente convexos, Tese, Instituto do Matemática Pura e Applicada, Rio de Janeiro, 1970; An. Acad. Brasil. Ci. 43 (1971). MR 41 \#7414.

5. A. Hirschowitz, Remarques sur les ouverts d'holomorphie d'un produit dénombrable de droites, Ann. Inst. Fourier (Grenoble) 19 (1969), fasc. 1, 219-229. MR 40 \#5892.

6. M. C. Matos, Sur l'enveloppe d'holomorphie des domaines de Riemann sur un produit dénombrable de droites, C. R. Acad. Sci. Paris Sér. A-B 271 (1970), A727-A728. MR 42 \#3554.

Department of Mathematics, University of Rochester, Rochester, New York 14627

Coppe, Universidade Federal do Rio de Janeiro, Caixa Postal 1191-ZC00, Rio de JANEIRO, GB., BRASIL

Current address: Instituto de Matemática, Universidade Federal do Rio de Janeiro, Caixa Postal 1835-ZC00, 20.000 Rio de Janeiro, GB., Brasil 